\title{
Long term follow up of patients with anterior myocardial infarction complicated by left ventricular thrombus in the thrombolytic era
}

\author{
Thomas Mooe, Dag Teien, Kjell Karp, Peter Eriksson
}

\begin{abstract}
Objectives-To examine the appearance and resolution of left ventricular thrombi and to study the relation between thrombus and mortality during long term follow up after anterior myocardial infarction. Design-Ninety nine consecutive patients were prospectively studied until the last included patient had been followed for one year. Streptokinase and aspirin were used routinely, anticoagulants only after a decision by the attending physician. Echocardiography was performed within $3 \mathrm{~d}$ of admission, before discharge, and after one, three, and 12 months.
\end{abstract}

Setting-Umeå University Hospital, a teaching hospital in Northern Sweden.

Main outcome measures-Left ventricular thrombus, segmental myocardial function, and mortality during follow up.

Results-Thirty patients (30\%) had a thrombus on discharge. One month, three months, and 12 months after hospital discharge, the thrombus had resolved in $81 \%$, $84 \%$, and $90 \%$ of the patients, respectively. The proportion of resolved thrombi at one month was high irrespective of whether anticoagulants were given $(10 / 11,91 \%)$ or not $(12 / 16,75 \%), P=0.4$. New thrombi appeared in 12 patients after discharge and resolution and reappearance of thrombi continued during the follow up period. Patients who developed a thrombus during the hospital stay ( $n=44,44 \%$ ) had more extensive myocardial dysfunction on discharge $(P<0.001)$ and significantly higher mortality during the follow up period than those without a thrombus $(23 \%$ v $7 \%$, P $<0.01$ ).

Conclusions-With routine thrombolytic and aspirin treatment of anterior myocardial infarction, left ventricular thrombi usually resolve during the first month after hospital discharge. Appearance and resolution of thrombi continue, however, in a

Table 1 Clinical and laboratory characteristics of patients with and without thrombus at discharge

\begin{tabular}{llll}
\hline & No thrombus, $n=69$ & Thrombus, $n=30$ & P value \\
\hline Age, mean (range) & $66 \cdot 2(41-85)$ & $67 \cdot 0(41-84)$ & NS \\
Men & $79 \%$ & $80 \%$ & NS \\
Previous myocardial infarction & $12 \%$ & $30 \%$ & $<0.05$ \\
Hypertension & $35 \%$ & $40 \%$ & NS \\
Diabetes & $16 \%$ & $10 \%$ & NS \\
Smokers & $31 \%$ & $11 \%$ & $<0.05$ \\
Peak LD1, mean (SD) & $15 \cdot 4(12 \cdot 3)$ & $20.8(9.5)$ & $<0.01$ \\
Peak CK, mean (SD) & $32.5(29.5)$ & $45.6(25 \cdot 2)$ & $<0.01$ \\
Peak CKMB, mean (SD) & $4 \cdot 1(3.8)$ & $5.4(3.1)$ & $<0.05$ \\
\hline
\end{tabular}

LD1, thermostable lactate dehydrogenase; $\mathrm{CK}$, creatine kinase. significant proportion of the patients during long term follow up. A left ventricular thrombus during the initial hospital stay is associated with high long term mortality.

(Heart 1996;75:252-256)

Keywords: myocardial infarction; left ventricular thrombosis; streptokinase; aspirin; mortality.

Patients with acute anterior myocardial infarction often develop a left ventricular thrombus during the hospital stay. ${ }^{1-3}$ Echocardiography is the preferred diagnostic method with a high sensitivity and specificity for ventricular thrombi. ${ }^{4}$ The finding of a thrombus has been associated with a more favourable prognosis, ${ }^{5}$ hypothetically by stabilising the left ventricular wall. Others, however, recommend anticoagulant treatment to achieve resolution of thrombi and to reduce the risk of embolisation. ${ }^{67}$ The recent introduction of thrombolytics and aspirin as a routine treatment in patients with acute myocardial infarction may, however, have changed the incidence of thrombi and their clinical significance. We therefore performed a study with serial echocardiographic examinations during the first year after anterior myocardial infarction. The aims of the study were to examine the appearance and disappearance of thrombi in consecutive patients discharged from hospital after an anterior myocardial infarct, to record embolic, cerebrovascular, and bleeding complications, and to study the long term prognosis in patients in whom a left ventricular thrombus was found during the hospital stay.

\section{Methods}

PATIENTS

Ninety nine consecutive patients surviving the hospital stay after an acute anterior myocardial infarction were included in the study. During the recruitment period three patients were excluded because of poor echocardiographic image quality and five patients died in hospital. Clinical and laboratory characteristics of the 99 study patients are shown in table 1 .

\section{DIAGNOSTIC STUDIES}

A diagnosis of anterior myocardial infarction was based on typical chest pain resistant to glyceryl trinitrate, a diagnostic rise in serum creatine kinase, and the following electrocardiographic findings: (1) the development of pathological $\mathrm{Q}$ waves in two or more precordial leads, or (2) ST segment elevation of $0.15 \mathrm{mV}$ in two 
Figure 1 Resolution and reappearance of left ventricular thrombi in patients with a thrombus at patients warge.

Echocardiographic

examinations during follow up were performed one, three, and 12 months after discharge. $T$, thrombus; 0 , no thrombus.

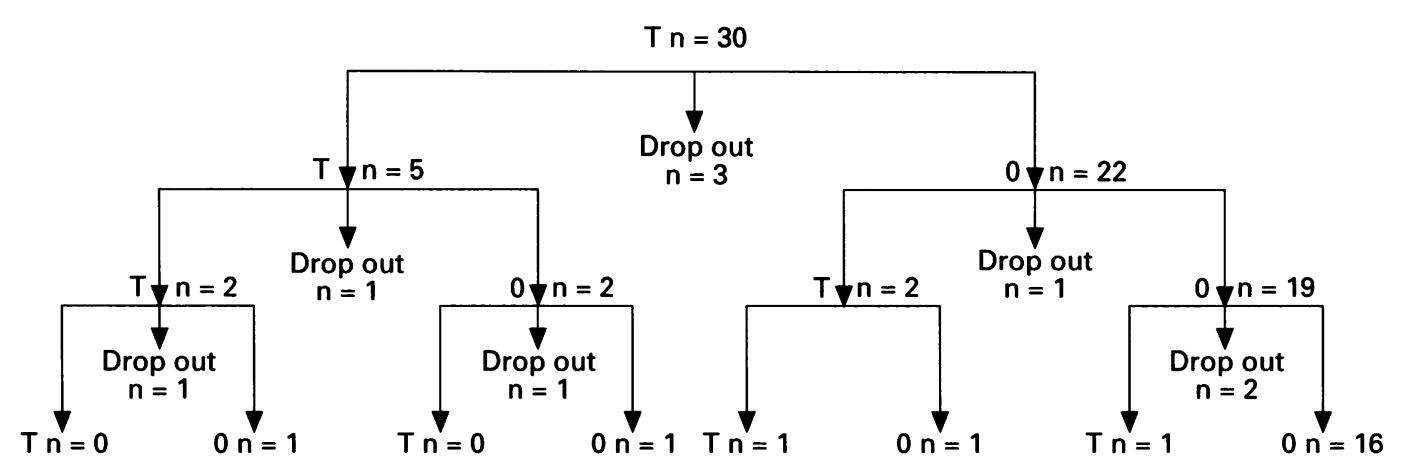

or more of leads $\mathrm{V} 1-\mathrm{V} 3$, or $0.1 \mathrm{mV}$ in two or more of leads V4-V6. Creatine kinase (CK) and creatine kinase-MB (CKMB) were measured on admission and at fixed hours four times daily. The time to peak CK from start of treatment was noted. Thermostable lactate dehydrogenase (LD1) was measured on admission and thereafter daily until the maximum level could be identified. Cross sectional echocardiographic examinations were performed within $3 \mathrm{~d}$ of admission, before discharge, and at one month, three months, and 12 months follow up. An Acuson XP-10 (Acuson Corporation) or a Vingmed CFM 750 (Vingmed Sound) equipped with $2 \cdot 5-5 \cdot 0 \mathrm{MHz}$ transducers were used. The examinations were recorded on a Panasonic AG 7330 video tape recorder and reviewed by two investigators unaware of treatment and previous findings. A left ventricular thrombus was defined as an echodense mass adjacent to an abnormally contracting myocardial segment. It should be distinguished from the underlying myocardium, have a clear thrombus-blood interface, and be seen in at least two transducer positions. ${ }^{48} \mathrm{~A} 16$ segment model was used for scoring the severity of segmental wall motion abnormalities according to the American Society of Echocardiography. ${ }^{9}$ The scoring scale ranged from 1 to 5 . A normally contracting or hyperkinetic segment was assigned a score of 1 , hypokinesis 2 , akinesis 3 , dyskinesis 4, and aneurysmal segments (diastolic deformation) 5 . The sum of all scores was calculated for each patient and used in the statistical analysis. The diagnosis of cerebral infarction required a sudden onset of focal neurological deficit and exclusion of haemorrhage by computerised tomography. Diuretics were used when there were clinical signs of heart failure according to the attending physician. An increased dose of peroral diuretics or the need for parenteral diuretics was thus used as a marker of congestive heart failure. All patients were prospectively followed and clinical events recorded until the last included patient had completed the 12 months echocardiographic examination. The mean follow up time was 23 months. The follow up time was more than one year for $91 \%(n=90)$ and more than two years for $48 \%(n=48)$ of the patients.

\section{TREATMENT}

Seventy four patients admitted within $6 \mathrm{~h}$ from onset of chest pain or with clinical signs of ongoing myocardial ischaemia and electrocardiographic evidence of an evolving myocardial infarct received streptokinase as an intravenous infusion of 1.5 million units over $1 \mathrm{~h}$. Heparin was not used. Twenty five patients did not receive streptokinase because of a long delay between onset of symptoms and hospital admission (20 patients), contraindication for thrombolytics (one patient), or for unknown reasons (four patients). Sixty six patients (67\%) without contraindications were given aspirin $160 \mathrm{mg}$ daily. Peroral anticoagulants were not routinely used, but 24 patients (24\%) were treated with warfarin at discharge, decided by the attending physician. No patient was given both warfarin and aspirin.

\section{STATISTICAL ANALYSIS}

Data were analysed with the STATISTICA $4 \cdot 0$ software modules (StatSoft Inc). Group data were expressed as mean (SD) for continuous variables and as rates for variables on a nominal scale. Differences between two means were assessed with $t$ test for unpaired data or the Mann Whitney U test when appropriate. Differences between proportions were analysed with the $\chi^{2}$ test, or for small sample sizes, by Fisher's exact test. Differences were considered significant for $P$ values less than $0 \cdot 05$. The relation between deteriorating left ventricular segmental function and clinical, laboratory, and echocardiographic data was examined by multiple logistic regression analysis. Kaplan-Meier survival curves were calculated for patients with and without a diagnosed thrombus during the hospital stay and survival times compared between groups with the log-rank test. The hazard ratio was calculated as a measure of relative survival.

\section{Results}

PATIENTS WITH A THROMBUS AT HOSPITAL DISCHARGE

The resolution and reappearance of left ventricular thrombi during the follow up period in patients with a thrombus present at discharge is shown in fig 1 . Most thrombi resolved during the first month. Resolution and reappearance of thrombi continued, however, during follow up, and no patient had a thrombus at all examinations. The proportion of patients with a thrombus at one month was $19 \%(5 / 27)$, at three months $16 \%(4 / 25)$, and at 12 months $10 \%$ $(2 / 21)$. There were nine dropouts in this group, of whom six died. Two patients did not participate in the three months examination, one of whom had a thrombus at 12 months. One 
Figure 2 Appearance and resolution of left ventricular thrombi in patients without thrombus at discharge. Echocardiographic examinations during follow up were performed one, three, and 12 months after discharge. $T$, thrombus, 0 , no thrombus.

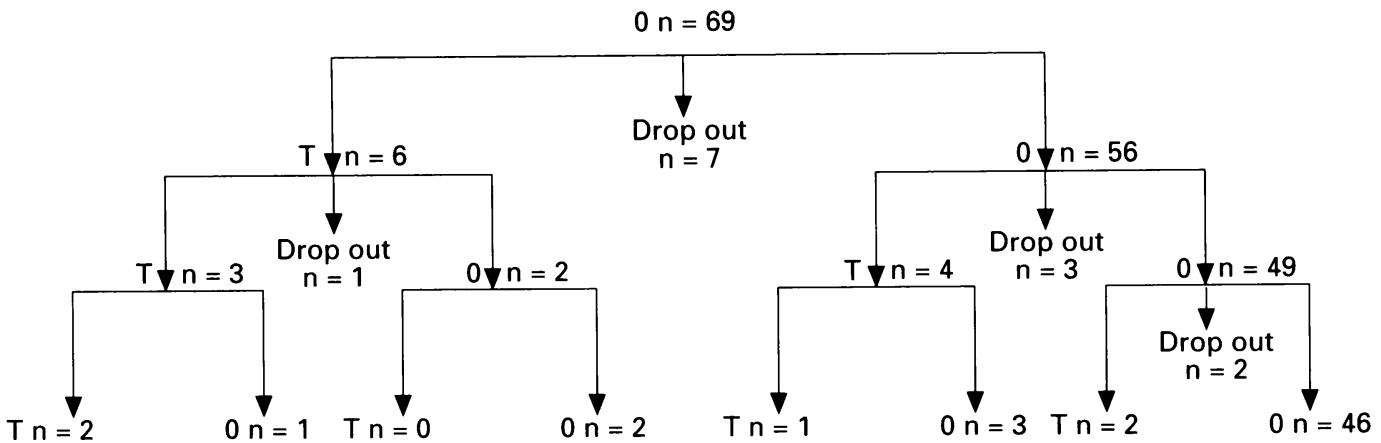

Table 2 Frequency of left ventricular thrombi at echocardiographic examinations during one year follow up after anterior myocardial infarction

\begin{tabular}{|c|c|c|c|c|c|c|c|c|}
\hline & \multicolumn{2}{|c|}{ Discharge } & \multicolumn{2}{|l|}{1 month } & \multicolumn{2}{|l|}{3 months } & \multicolumn{2}{|l|}{1 year } \\
\hline & $\begin{array}{l}T \\
\%(n)\end{array}$ & $\begin{array}{l}0 \\
\%(n)\end{array}$ & $\begin{array}{l}T \\
\%\left(n ; n^{l}\right)\end{array}$ & $\begin{array}{l}0 \\
\%(n)\end{array}$ & $\begin{array}{l}T \\
\%\left(n ; n^{l}\right)\end{array}$ & $\begin{array}{l}0 \\
\%(n)\end{array}$ & $\begin{array}{l}T \\
\%\left(n ; n^{\prime}\right)\end{array}$ & $\begin{array}{l}0 \\
\%(n)\end{array}$ \\
\hline All patients & $30(30)$ & $70(69)$ & $12(11 ; 6)$ & $88(78)$ & $13(11 ; 7)$ & $87(74)$ & $10(8 ; 5)$ & $90(76)$ \\
\hline Streptokinase & $31(23)$ & $69(51)$ & $11(7 ; 4)$ & $89(59)$ & $16(10 ; 6)$ & $84(53)$ & $8(5 ; 3)$ & $92(57)$ \\
\hline No streptokinase & $28(7)$ & $72(18)$ & $17(4 ; 2)$ & $83(19)$ & $5(1 ; 1)$ & $95(21)$ & $14(3 ; 2)$ & $86(19)$ \\
\hline Warfarin & $54(13)$ & $46(11)$ & $6(1 ; 0)$ & $94(17)$ & $12(2 ; 0)$ & $88(15)$ & $0(0)$ & $100(9)$ \\
\hline No warfarin & $23(17)$ & 77 (58) & $14(10 ; 6)$ & $86(61)$ & $13(9 ; 7)$ & $87(59)$ & $11(8 ; 5)$ & $89(67)$ \\
\hline
\end{tabular}

$\mathrm{T}$, thrombus; 0 , no thrombus; $\mathrm{n}$, number of patients; $\mathrm{n}^{1}$, number of patients in the subgroup with new thrombi formed after discharge.

patient did not participate in the 12 months examination.

\section{PATIENTS WITHOUT A THROMBUS AT HOSPITAL DISCHARGE}

Figure 2 shows the appearance and resolution of left ventricular thrombi during follow up in patients without a thrombus at discharge. A new thrombus was found in some patients at every interval of follow up examination, including 12 months after discharge. The proportion of patients with a thrombus after one month was $10 \%(6 / 62)$, three months $12 \%(7 / 58)$, and 12 months $9 \%(5 / 57)$. There were 12 drop outs in this group, of whom five died. One patient went through a mitral valve replacement with a complicated postoperative period. Two older patients did not want to participate in further follow up after discharge. Four patients did not undergo the one month or the three months examination. None of them had a thrombus at the remaining examinations.

Table 2 shows the prevalence of left ventricular thrombi during follow up in all patients in relation to treatment with streptokinase and warfarin. After a rapid decrease in prevalence of thrombi during the first month, no significant difference between the groups was seen during further follow up. The proportion of resolved thrombi in surviving patients one month after discharge was $85 \%(17 / 20)$ in those given streptokinase and $71 \%(5 / 7)$ in those not given streptokinase. Similarly, the proportion of resolved thrombi was $91 \%(10 / 11)$ in those given warfarin and $75 \%(12 / 16)$ in those not given warfarin $(P=0.4)$. After hospital discharge, new thrombi appeared in patients given streptokinase $(20 \%, 9 / 46)$ as well as in patients not given streptokinase $(19 \%, 3 / 16)$, while no new thrombi appeared in patients on warfarin $(0 / 7)$. New thrombi appeared in $22 \%(12 / 55)$ of the patients who were not given warfarin $(\mathbf{P}$ $=0 \cdot 3$ ). Left ventricular segmental dysfunction at discharge was significantly more extensive in patients with a thrombus during the hospital stay compared with those without a thrombus (segmental score 25.7 v 21.4, P < 0.001), fig 3 . Patients with a thrombus also had more extensive segmental dysfunction at the follow up examinations.

Twenty nine patients (35\%) had more extensive segmental myocardial dysfunction at the one year follow up compared with the examination at discharge. The maximum level of $\mathrm{LD} 1$ was the most important predictor of deteriorating left ventricular function. An increase of 1 SD (11.7) in LD1 was associated with a fourfold increase in the risk of further impairment of left ventricular function (odds ratio $4 \cdot 2,95 \%$ confidence interval 1.5 to $11 \cdot 8$ ). Fourteen patients died during the follow up period, 10 of whom had a thrombus during the hospital stay. Eight of these deaths were cardiovascular (sudden death, myocardial infarction, congestive

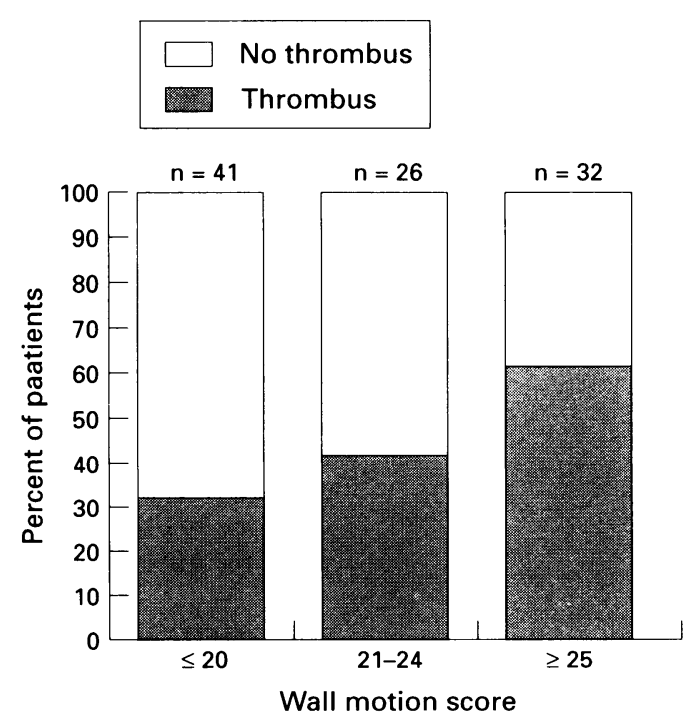

Figure 3 Proportion of patients with thrombus at different levels of wall motion score. 


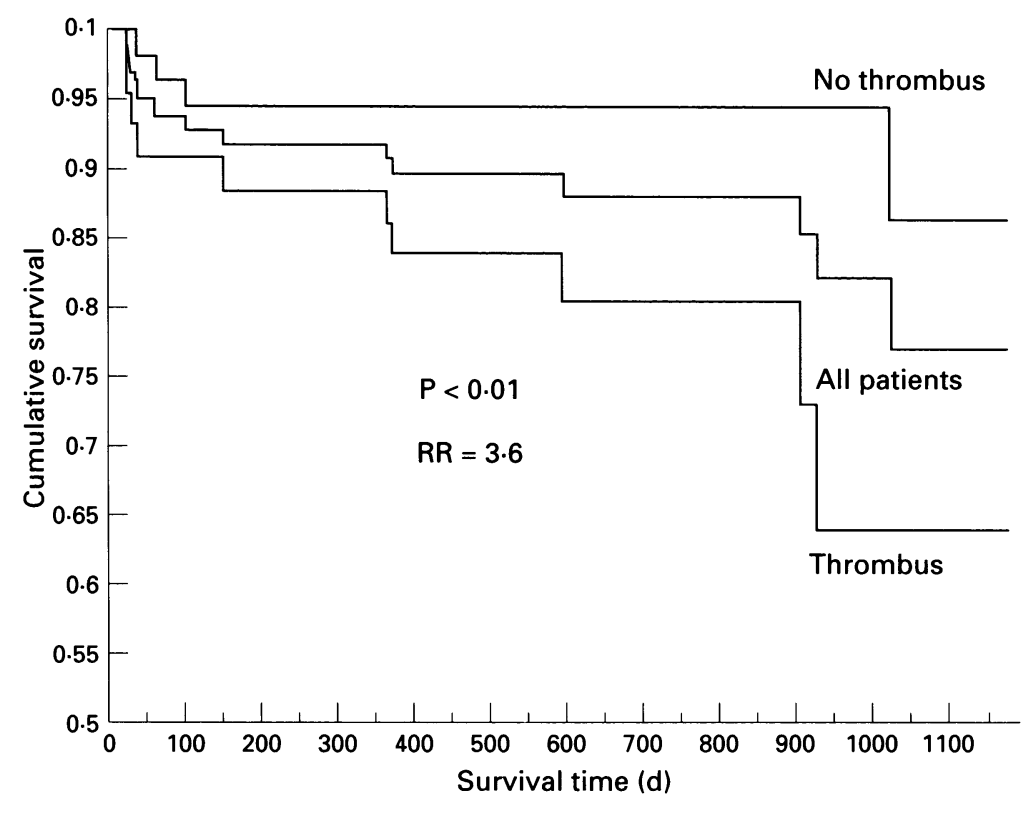

Figure 4 Cumulative survival during follow up for the entire study group and in patients with ( $n=44,10$ deaths) and without ( $n=55,4$ deaths) a left ventricular thrombus during the hospital stay. $R R$, relative risk. non-invasive nature. ${ }^{4}$ The method is accurate, with a sensitivity ranging between $77 \%$ and $100 \%$ and a specificity ranging between $8 \%$ and $100 \%$ when compared with findings at surgery or necropsy, or with platelet imaging. The accuracy of echocardiography is dependent of the image quality, and small thrombi, below the level of detection, may be present in areas of disturbed flow conditions. Potential diagnostic errors may, however, be minimised by strict adherence to echocardiographic criteria. ${ }^{10}$ In this study we showed that the majority of left ventricular thrombi formed during the course of an anterior myocardial infarction resolve during the first three months after discharge $(21 / 25,84 \%)$. The proportion of thrombi reported to resolve during long term follow up in most previous studies was smaller, ranging between $20 \%$ and $58 \% .^{235}$ In one study oral anticoagulants were used routinely and resolution of thrombi were reported in $33 \%(4 / 14)$ after 12 months of follow up. ${ }^{1}$ Treatment of left ventricular thrombi with oral anticoagulants has been examined in a controlled trial, and resolution of thrombi occurred in $59 \%$ and $88 \%$ of the patients after three and 12 months. ${ }^{11}$ The high proportion of thrombi resolving during follow up in our study may be associated with new treatment routines after myocardial infarction. Aspirin reduces thromboembolic complications in patients with atrial fibrillation $^{12}$ and may modify platelet deposition in some patients with left ventricular thrombi. ${ }^{13}$ The use of thrombolytics and aspirin may hypothetically influence the thrombus structure and make it more susceptible to endogenous thrombolysis. Thirty patients had a thrombus at discharge (fig 1) and in $29 \%(12 / 42)$ a new thrombus appeared during follow up (fig 2). In two previous reports the proportion of thrombi appearing after discharge was similar, $31 \%$ and $32 \%$, respectively. ${ }^{13}$ Thrombi formed after discharge constituted a large proportion of thrombi diagnosed after one month $(55 \%)$ three months (64\%), and one year $(62 \%)$. Warfarin treatment seemed to prevent the formation of new thrombi after discharge, but the number of patients given warfarin was small. The dynamic process of resolution and reappearance of thrombi makes it difficult to use echocardiography to identify patients at risk for thromboembolic complications. If a thrombus has resolved at one examination, a new thrombus may well appear later on. Early thrombus formation (during the hospital period) has been reported to be associated with a high mortality. $^{214} \mathrm{We}$ found that a left ventricular thrombus diagnosed during the hospital stay is a marker for high mortality after discharge. In one previous study, early in-hospital mortality was higher in patients without a thrombus. ${ }^{5}$ However, several patients did not survive long enough for a ventricular thrombus to form. ${ }^{15}$ In the same study no difference in myocardial infarct size was found in patients with and without a thrombus, in contrast to our findings and to several previous reports ${ }^{16-18}$ where patients with a thrombus had more extensive myocardial dysfunction. Three cerebral ischaemic events were recorded during the follow up period. The
Echocardiography is the method of choice for the serial identification of ventricular thrombi because of its wide availability, low cost, and 
episode of amaurosis fugax was probably associated with a right sided internal carotid stenosis, whereas the other two events may have been embolic. It is, however, very difficult to differentiate between embolic and thrombotic events in patients with advanced atherosclerotic disease. ${ }^{19}$ The incidence of cerebral embolisation was approximately $1 \%$ in large scale postinfarction trials investigating thrombolytic therapy. ${ }^{2021}$ Two serious bleeding episodes occurred, a reminder that warfarin may be associated with fatal complications, reported to occur in $1 \%$ to $4.8 \% .{ }^{22}$ We conclude that after treatment of anterior myocardial infarction with thrombolytics and aspirin most left ventricular thrombi resolves during the first month after discharge, but formation and resolution of thrombi continues during further follow up. The dynamic process of left ventricular thrombus formation and resolution makes it difficult to use echocardiography to identify patients at risk for thromboembolic complications. The formation of a thrombus during the hospital stay is associated with high mortality after discharge. Warfarin may prevent formation of new thrombi after hospital discharge, but the low incidence of cerebral embolisation after myocardial infarction and the risk of serious bleedings during warfarin treatment should be considered before instituting treatment with anticoagulants.

This study was supported by grants from the Medical Faculty, Umeå University and the Joint Committee of the Northern Swedish Health Care Region.

1 Visser CA, Kan G, Meltzer RS, Lie KI, Durrer D. Longterm follow-up of left ventricular thrombus after acute myocardial infarction. A two-dimensional echocardiographic study in 96 patients. Chest 1984;86:532-6.

2 Spirito P, Bellotti P, Chiarella F, Domenicucci S, Sementa A, Vecchio C. Prognostic significance and natural history A, Vecchio C. Prognostic significance and natural history
of left ventricular thrombi in patients with acute anterior of left ventricular thrombi in patients with acute anterior
myocardial infarction: a two-dimensional echocardiomyocardial infarction: a two-dimensiona

3 Keren A, Goldberg S, Gottlieb S, et al. Natural history of left ventricular thrombi: their appearance and resolution in the posthospitalization period of acute myocardial infarction. F Am Coll Cardiol 1990;15:790-800.

4 Ezekowitz MD. Identifying left ventricular thrombi. Clin Cardiol 1990;13(suppl 6):VI31-3.

5 Nihoyannopoulos P, Smith GC, Maseri A, Foale RA. The natural history of left ventricular thrombus in myocardial infarction: a rationale in support of masterly inactivity. $f$ Am Coll Cardiol 1989;14:903-11.
6 Fuster V, Halperin JL. Left ventricular thrombi and cerebral embolism. N Engl f Med 1989;320:392-4.

7 Vaitkus PT, Barnathan ES. Embolic potential, prevention and management of mural thrombus complicating anteand management of mural thrombus complicating anterior myocardial infarction:

8 Asinger RW, Mikell FL, Sharma B, Hodges M Observations on detecting left ventricular thrombus with two dimensional echocardiography: emphasis on avoidance of false positive diagnoses. Am $\mathcal{f}$ Cardiol 1981 47:145-56.

9 Schiller NB, Shah PM, Crawford M, et al. Recommendations for quantitation of the left ventricle by twodimensional echocardiography. American Society of Echocardiography Committee on Standards, Subcommittee on Quantitation of Two-Dimensional Echocardiograms. $¥ \mathrm{Am}$ Soc Echocardiogr 1989;2:358-67.

10 Stratton JR, Lighty G, Pearlman AS, Ritchie JL. Detection of left ventricular thrombus by two-dimensional echocardiography: sensitivity, specificity, and causes of uncerdiography: sensitivity, specificity,

11 Tramarin R, Pozzoli M, Febo O, et al. Two-dimensional echocardiographic assessment of anticoagulant therapy in left ventricular thrombosis early after
infarction. Eur Heart $¥$ 1986;7:482-92.

12 Stroke prevention in atrial fibrillation investigators Warfarin versus aspirin for prevention of thromboembolism in atrial fibrillation: stroke prevention in atrial fibrillation II study. Lancet 1994;343:687-91.

13 Stratton JR, Ritchie JL. The effects of antithrombotic drug in patients with left ventricular thrombi: assessment with indium-111 platelet imaging and two-dimensiona echocardiography. Circulation 1984;69:561-8.

14 Domenicucci S, Chiarella F, Bellotti P, Lupi G, Scarsi G, Vecchio C. Early appearance of left ventricular thrombi after anterior myocardial infarction: a marker of higher in-hospital mortality in patients not treated with antithrombotic drugs. Eur Heart $\mathcal{f} 1990 ; 11: 51-8$.

15 Halperin JL, Fuster V. Left ventricular thrombus and stroke after myocardial infarction: toward prevention or perplexity? f Am Coll Cardiol 1989;14:912-4.

16 Kupper AJ, Verheugt FW, Peels CH, Galema TW, Roos JP. Left ventricular thrombus incidence and behavio studied by serial two-dimensional echocardiography in acute anterior myocardial infarction: left ventricular wal motion, systemic embolism and oral anticoagulation. $\mathcal{f}$ Am Coll Cardiol 1989;13:1514-20.

17 Vecchio C, Chiarella F, Lupi G, Bellotti P, Domenicucci S Left ventricular thrombus in anterior acute myocardial infarction after thrombolysis. A GISSI-2 connected study. Circulation 1991;84:512-9.

18 Kontny F, Dale J, Hegrenaes L, Lem P, Soberg T, Morstol $T$. Left ventricular thrombosis and arterial embolism after thrombolysis in acute anterior myocardial infarction: predictors and effects of adjunctive antithrombotic therapy. Eur Heart $\mathcal{f} 1993 ; 14: 1489-92$.

19 Cardiogenic brain embolism. The second report of the Cerebral Embolism Task Force. Arch Neurol 1989;46: 727-43.

20 GISSI-2: a factorial randomised trial of alteplase versus streptokinase and heparin versus no heparin among 12,490 patients with acute myocardial infarction. 12,490 patients with acute myocardial infarction. Gruppo Italiano per lo Studio della Sopra

21 ISIS-3: a randomised comparison of streptokinase vs tissue plasminogen activator vs anistreplase and of aspirin plus heparin vs aspirin alone among 41,299 cases of suspected acute myocardial infarction. ISIS-3 (Third International Study of Infarct Survival) Collaborative Group. Lance 1992;339:753-70.

22 Levine MN, Raskob G, Hirsh J. Hemorrhagic complications of long-term anticoagulant therapy. Chest 1986; 89(suppl 2):16-25S 